\title{
The HDF1 Histone Deacetylase Gene Is Important for Conidiation, Sexual Reproduction, and Pathogenesis in Fusarium graminearum
}

\author{
Yimin Li, ${ }^{1,2}$ Chenfang Wang, ${ }^{1}$ Wende Liu, ${ }^{2}$ Guanghui Wang, ${ }^{1}$ Zhensheng Kang, ${ }^{1}$ H. Corby Kistler, ${ }^{3}$ \\ and Jin-Rong $X u^{1,2}$ \\ ${ }^{1}$ College of Plant Protection and Shaanxi Key Laboratory of Molecular Biology for Agriculture, Northwest A\&F University, \\ Yangling, Shanxi 712100, China; ${ }^{2}$ Department of Botany and Plant Pathology, Purdue University, West Lafayette, IN 47907, \\ U.S.A.; ${ }^{3}$ United States Department of Agriculture-Agricultural Research Service, Cereal Disease Laboratory, University \\ of Minnesota, St. Paul, MN 55108, U.S.A.
}

Submitted 11 October 2010. Accepted 23 November 2010.

Head blight caused by Fusarium graminearum is an important disease of wheat and barley. Its genome contains chromosomal regions with higher genetic variation and enriched for genes expressed in planta, suggesting a role of chromatin modification in the regulation of infection-related genes. In a previous study, the $F$ TL1 gene was characterized as a novel virulence factor in the head blight fungus. FTL1 is homologous to yeast $S I F 2$, which is a component of the Set3 complex. Many members of the yeast Set3 complex, including Hos2 histone deacetylase (HDAC), are conserved in $F$. graminearum. In this study, we characterized the $H D F 1$ gene that is orthologous to $H O S 2$. HDF1 physically interacted with FTL1 in yeast two-hybrid assays. Deletion of $H D F 1$ resulted in a significant reduction in virulence and deoxynivalenol (DON) production. The $\Delta$ hdf1 mutant failed to spread from the inoculation site to other parts of wheat heads or corn stalks. It was defective in sexual reproduction and significantly reduced in conidiation. Expression of $H D F 1$ was highest in conidia in comparison with germlings and hyphae. Deletion of $H D F 1$ also resulted in a $60 \%$ reduction in HDAC activity. Microarray analysis revealed that 149 and 253 genes were down- and upregulated, respectively, over fivefold in the $\Delta h d f 1$ mutant. Consistent with upregulation of putative catalase and peroxidase genes, the $\Delta h d f 1$ mutant was more tolerant to $\mathrm{H}_{2} \mathrm{O}_{2}$ than the wild type. Deletion of the other two class II HDAC genes had no obvious effect on vegetative growth and resulted in only a minor reduction in conidiation and virulence in the $\Delta h d f 2$ mutant. Overall, our results indicate that $H D F 1$ is the major class II HDAC gene in $F$. graminearum. It may interact with FTL1 and function as a component in a well-conserved HDAC complex in the regulation of conidiation, DON production, and pathogenesis.

Fusarium head blight (FHB) or scab is one of the most important diseases of wheat and barley (Bai and Shaner 2004;

\section{Y. Li and C. Wang contributed equally to this work.}

Corresponding author: J.-R. Xu; E-mail: jinrong@purdue.edu; Telephone: +1.765.496.6918; Fax: +1.765.496.6918.

* The $\boldsymbol{e}$-Xtra logo stands for "electronic extra" and indicates that four supplementary tables and five supplementary figures are published online. Figures 3, 4, 5, and 10 also appear in color online.
Goswami and Kistler 2004). Fusarium graminearum (teleomorph Gibberella zeae) is the major causal agent of head blight in North America and other parts of the world. Plant infection is initiated when ascospores (the primary inoculum) ejected into the air by the pathogen (Mitter et al. 2006; Trail et al. 2005) are dispersed and deposited on flowering wheat or barley heads, which are susceptible to infection from the beginning of anthesis to the dough stage of kernel development. After initial colonization, the pathogen can spread from the infection site to other florets (Brown et al. 2010) and cause severe yield losses under favorable environmental conditions. In addition, $F$. graminearum produces harmful mycotoxins, such as deoxynivalenol (DON) and zearalenone. DON is also phytotoxic and an important virulence factor in the wheat scab fungus (Desjardins 2003; Proctor et al. 1995).

The $F$. graminearum genome has no active transposable elements and contains less than $0.5 \%$ repetitive sequences (Cuomo et al. 2007). Another unique feature of its genome is the presence of regions enriched for genes that are unique or highly expressed during plant infection (Cuomo et al. 2007). Some of these genes are known to be involved in fungus-plant interactions, suggesting that chromatin structure and modifications play a role in regulating infection-related genes. Reversible acetylation and deacetylation are common forms of histone modifications mediated by histone acetyltransferases (HAT) and histone deacetylases (HDAC). Classical HDAC include class I and class II HDAC that are sensitive to inhibition by trichostatin A and share similarity to yeast Rpd3 and Hda1, respectively (Yang and Grégoire 2005). In several fungi, histone modifications have been implicated in regulating genes important for pathogenicity, stress response, and secondary metabolism (Baidyaroy et al. 2001; Ding et al. 2009; Palmer et al. 2008; Tribus et al. 2010). In the corn pathogen Cochliobolus carbonum, the HDCl HDAC gene is an important pathogenicity factor (Baidyaroy et al. 2001). In Aspergillus nidulans, the Rpd3-like HDAC is required for hyphal growth and conidiation (Tribus et al. 2010). HdaA is a class II HDAC important for the biosynthesis of sterigmatocystin and production of conidia in A. fumigatus (Lee et al. 2009). In the rice blast fungus Magnaporthe oryzae, the Tig1 HDAC complex is essential for invasive growth and blast lesion development (Ding et al. 2010).

In $F$. graminearum, the $\Delta f t l 1$ mutant was defective in colonizing wheat heads and causing typical head blight symptoms (Ding et al. 2009). FTL1 is homologous to yeast SIF2, which 
is a component of the Set3 complex regulating genes important for late stages of sporulation (Cohen et al. 2008; Wang et al. 2002). Many members of the yeast Set 3 complex, including the Hos2 HDAC and Snt1 protein, are conserved in $F$. graminearum and may play a role in regulating plant infection and DON production. In this study, we identified and characterized the $F$. graminearum ortholog of yeast HOS2 (named $H D F 1)$. Hdf1 physically interacted with Ftl1 in yeast twohybrid assays. Mutants deleted of the $H D F 1$ gene were significantly reduced in virulence and DON production. The $\Delta h d f 1$ mutant was defective in sexual reproduction and significantly reduced in conidiation and HDAC activity. Deletion of the other two class II HDAC genes had no effect on vegetative growth. The $\Delta h d f 3$ mutant was normal and the $\Delta h d f 2$ mutant had only a minor reduction in conidiation and virulence. Overall, our results indicate that Hdf1 is the major class II HDAC gene in $F$. graminearum and is involved in the regulation of conidiation, DON production, and plant infection.

\section{RESULTS}

\section{Identification of the HOS2 ortholog}

in the $F$. graminearum genome.

The genome of $F$. graminearum contains three class II HDAC genes (FGSG_01353, FGSG_04324, and FGSG_05636), which are orthologous to yeast $H O S 2, H D A 1$, and $H O S 3$, respectively. Phylogenetic analysis indicates that these three class II HDAC genes are well conserved in other filamentous fungi. The HOS 2 ortholog was designated HDAC in F. graminearum 1 $(H D F 1)$. It shares $78 \%$ identity at the amino acid level with $C$. carbonum HDC1, which is the first HDAC gene known to be important for fungal pathogenicity (Baidyaroy et al. 2001). Hdf1 is also homologous to Hos2 in M. oryzae (Ding et al. 2010) and HosA in A. nidulans (Graessle et al. 2000).

Because Hos2 forms an HDAC complex with Sif2 in yeast, we constructed the FTL1 bait and HDF1 prey vectors. Growth on SD-His-Trp-Ura plates and LacZ activities were observed in transformants expressing both constructs (Fig. 1), indicating that Hdf1 interacts with Ftl1 in yeast two-hybrid assays.

\section{HDF1 is not essential for vegetative growth.}

To determine its function in $F$. graminearum, we generated the $\Delta h d f 1$ mutant by the split-marker approach (Fig. 2A). Putative $H D F 1$ deletion mutants were identified by polymerase chain reaction (PCR) and confirmed by Southern blot hybridization. When probed with the $H D F 1$ fragment amplified with

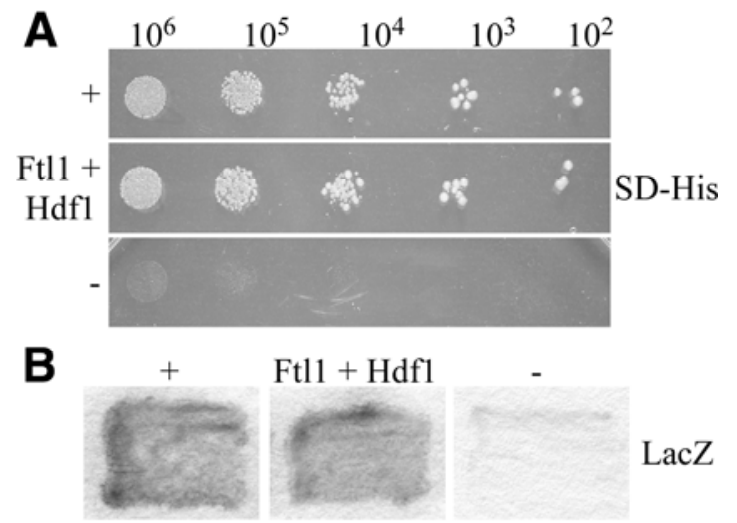

Fig. 1. Yeast two-hybrid assays for the interaction between FTL1 and $H D F 1$. Transformants expressing the HDF1 bait and FTL1 prey constructs were assayed for growth on SD-Leu-Trp-His plates (SD-His) and $\beta$-galactosidase (LacZ) activities. Positive $(+)$ and negative $(-)$ controls were from the Stratagene HybridZap library construction kit. primers F5 and R6, the wild-type PH-1 had a 5.6-kb HindIII band but transformants YM1 through YM6 lacked hybridization signals (Fig. 2B). When hybridized with a fragment of the hygromycin phosphotransferase $(h p h)$ gene, the wild type had no hybridization signals. The $\Delta h d f 1$ mutants YM1, YM2, YM5, and YM6 (Table 1) had a 5.2-kb band resulting from the gene replacement event (Fig. 2B). All of the $\Delta h d f 1$ mutants had the same phenotype, although only data for YM1 were described below. The $\Delta h d f 1$ mutant was only slightly reduced in vegetative growth on potato dextrose agar (PDA) or complete medium (CM) plates but was reduced over 10-fold in conidiation (Table 2). In comparison with the wild type, colonies formed by the $\Delta h d f 1$ mutant on PDA appeared to be less pigmented (Fig. 2C).

\section{The $\Delta h d f 1$ mutant is significantly reduced in virulence.}

In wheat heads inoculated with the $\Delta h d f 1$ mutant, only the inoculated spikelets developed typical disease symptoms (Fig. 3A) 14 days postinoculation (dpi). Under the same conditions, the wild-type strain was able to spread from the inoculation sites to nearby spikelets (Fig. 3A). The average disease index for YM1 and PH-1 was 1.1 and 11.6, respectively (Table 2), indicating that the $\Delta h d f l$ mutant was defective in disease spreading and colonization of wheat heads but not in the initial infection. Because $F$. graminearum also is a pathogen on corn, we conducted infection assays with corn stalks. In stalk rot assays, the mutant caused only limited discoloration at the inoculation sites 14 dpi. Under the same conditions, extensive discoloration was observed in corn stalks inoculated with PH-1 (Fig. $3 \mathrm{~B}$ ), indicating the spreading and colonization in the pith by the wild type.

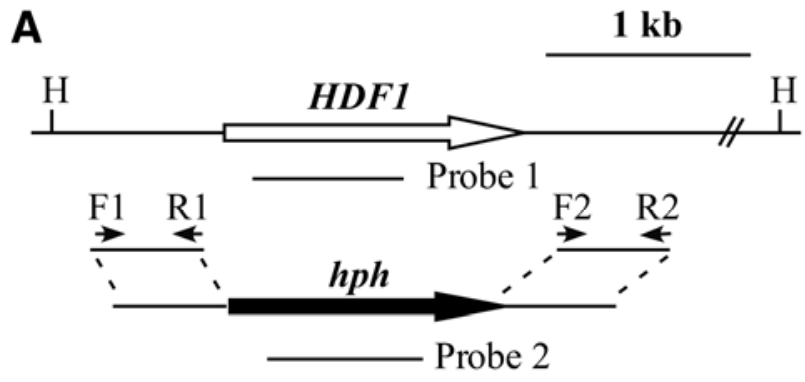

B Probe 1

Probe 2

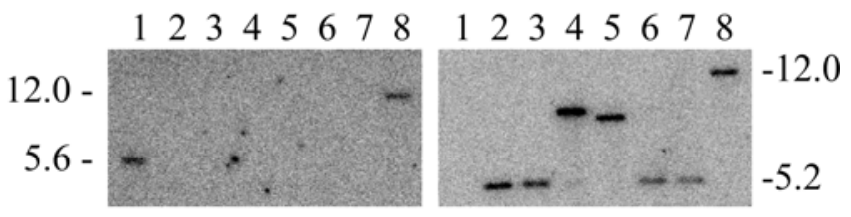

C

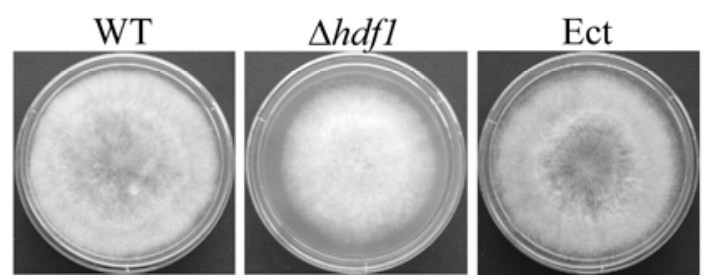

Fig. 2. $H D F 1$ gene and deletion mutants. A, HDF1 locus and gene replacement construct. The HDF1 and hygromycin phosphotransferase (hph) genes are marked with empty and black arrows, respectively. H, HindIII. $\mathbf{B}$, Southern blots of HindIII-digested DNA hybridized with fragments of the HDF1 (probe 1, left) and $h p h$ (probe 2, right) genes. Lanes 1 to 8: DNA samples from the wild type and transformants YM1-YM7. C, Colonies of the wild type (WT), $\Delta h d f 1$ mutant (YM1), and an ectopic transformant (Ect) on potato dextrose agar plates. 
To confirm that the defects of the $\Delta h d f 1$ mutant in plant infection directly resulted from the gene deletion event, we cloned the wild-type $H D F$ gene (containing $1.5 \mathrm{~kb}$ of promoter and $0.6-\mathrm{kb}$ terminator regions) and transformed it into protoplasts of the $\Delta h d f 1$ mutant YM1. Geneticin-resistant transformants, including YM11 (Table 1), were isolated and confirmed by PCR. Virulence of YM11 in infection assays with wheat heads (Table 2) and corn stalks (data not shown) were normal.

The $\Delta h d f 1$ mutant is defective in colonizing wheat rachis.

To further determine the function of $H D F 1$ in plant infection, we examined the infection processes by scanning and transmission electron microscopy (SEM and TEM). At $24 \mathrm{~h}$ postinoculation (hpi), both wild-type and mutant strains directly penetrated the wheat epidermis (Fig. 4A). Fungal growth was observed in lemma tissues 48 hpi (Fig. 4B), indicating that the $\Delta h d f 1$ mutant was normal in the initial infection processes. However, HDFl appeared to be essential for spreading from the inoculated spikelet to the rachis and nearby spikelets. By $120 \mathrm{hpi}$, fungal growth was not observed in the rachis below or above the spikelets inoculated with the $\Delta h d f 1$ mutant (Fig. 4C), whereas the wild type had colonized the vascular and other tissues of the rachis. Abundant intracellular hyphae were observed in rachis tissues (Fig. 4C). These observations were consistent with reduced virulence of the mutant (Table 2).

We then transformed the $\mathrm{P}_{\mathrm{RP} 27}-\mathrm{eGFP}$ construct $\mathrm{pRM} 7$ (Mehrabi et al. 2008) into the wild-type strain PH-1 and $\Delta h d f 1$ mutant YM1. The resulting transformants RM7PH1 and YM10 (Table 1) had strong green fluorescent protein (GFP) signals (data not shown) and were used to inoculate flowering wheat heads. At 5 dpi, fungal growth was examined with an epifluorescence microscope after removing the inoculated spikelets. Extensive GFP signals were observed in the rachis of wheat heads inoculated with RM7PH1 (Fig. 5). In contrast, YM10 failed to colonize the rachis tissues. GFP signals were restricted to the sites where the colonized spikelets were attached (Fig. 5), further indicating that the $\Delta h d f 1$ mutant was defective in spreading from the inoculation site to the wheat rachis.

\section{Deletion of $H D F 1$ blocks sexual reproduction.}

$F$. graminearum is a homothallic fungus. On carrot agar plates, the wild type produced abundant perithecia 2 to 3 weeks after self fertilization. Many perithecia produced ascospore cirrhi that emerged through the ostiole (Fig. 6A). Under the same conditions, no perithecia were formed by the $\Delta h d f 1 \mathrm{mu}-$ tant. Close examination revealed that the $\Delta h d f 1$ mutant formed numerous sporodochia as tufts on carrot agar plates (Fig. 6). Abundant conidiophores and conidia were produced by the mutant in the sporodochia (Fig. 6B). These results indicate that $H D F 1$ is essential for sexual reproduction in self crosses of $F$. graminearum. Production of sporodochia instead of perithecia on mating plates suggests that the $\Delta h d f l$ mutant is defective in the proper regulation of sexual and asexual reproduction.

\section{HDAC activity is reduced in the $\Delta h d f 1$ mutant.}

To confirm the biochemical function of the product of the HDF 1 gene, we isolated proteins from vegetative hyphae of the $\Delta h d f 1$ mutant. HDAC activities were assayed with the colorimetric HDAC assay kit (as described below). HDAC activity in the $\Delta h d f 1$ mutant was reduced approximately $60 \%$ compared with the wild type (Fig. 7), indicating that $H D F 1$ is the major type II HDAC gene in $F$. graminearum.

\section{Expression and localization of the HDF1-eGFP fusion construct.}

An $H D F 1$-eGFP fusion construct was transformed into the $\Delta h d f 1$ mutant YM1 (Table 1). The resulting transformant, YM9 (Table 1), had no or very weak GFP signals in the cytoplasm

Table 1. Strains used in this study

\begin{tabular}{|c|c|c|}
\hline Name & Genotype & References \\
\hline PH-1 & Wild type & Cuomo et al. 2007 \\
\hline 11622 & nit1 mutant & Hou et al. 2002 \\
\hline YM1 & $\Delta h d f 1$ mutant & This study \\
\hline YM2 & $\Delta h d f 1$ mutant & This study \\
\hline YM5 & $\Delta h d f 1$ mutant & This study \\
\hline YM6 & $\Delta h d f 1$ mutant & This study \\
\hline YM8 & Ectopic transformant of the $H D F 1$ knockout construct & This study \\
\hline YM9 & Transformant of YM1 expressing the $H D F 1$-eGFP fusion & This study \\
\hline YM10 & Transformant of YM1 expressing the RP27-eGFP construct & This study \\
\hline YM11 & Complemented transformant of YM1 $(\Delta h d f 1 / H D F 1)$ & This study \\
\hline YM12 & $\Delta h d f 3$ deletion mutant & This study \\
\hline YM13 & $\Delta h d f 2$ deletion mutant & This study \\
\hline YM14 & Ectopic transformant of the $H D F 3$ knockout construct & This study \\
\hline YM15 & Ectopic transformant of the $H D F 2$ knockout construct & This study \\
\hline YM16 & $\Delta h d f 1 \Delta h d f 2$ double mutant & This study \\
\hline YM17 & $\Delta h d f 1 \Delta h d f 3$ double mutant & This study \\
\hline RM7PH1 & Transformant of YM1 expressing the RP27-eGFP construct & This study \\
\hline
\end{tabular}

Table 2. Vegetative growth, conidiation, and virulence of Fusarium graminearum strains ${ }^{\mathrm{a}}$

\begin{tabular}{|c|c|c|c|c|}
\hline Strain & Growth rate $(\mathrm{mm} / \mathrm{day})^{\mathrm{b}}$ & Conidiation $\left(\times 10^{4} / \mathrm{ml}\right)^{c}$ & Disease index & DON $(p p m)^{e}$ \\
\hline PH-1 (wild type) & $11.1 \pm 0.3$ & $124.0 \pm 6.7$ & $11.6 \pm 5.6$ & $913.8 \pm 151.2$ \\
\hline YM1 $(\Delta h d f 1)$ & $8.0 \pm 0$ & $9.8 \pm 5.3$ & $1.1 \pm 4.8$ & $617.5 \pm 50.7$ \\
\hline$Y M 13(\Delta h d f 2)$ & $10.7 \pm 1.3$ & $52.0 \pm 4.5$ & $7.5 \pm 8.9$ & $915.0 \pm 258.5$ \\
\hline YM12 ( $\Delta h d f 3)$ & $10.3 \pm 1.1$ & $104.0 \pm 4.6$ & $10.9 \pm 7.9$ & $358.3 \pm 217.7$ \\
\hline YM8 (ectopic) & $10.3 \pm 0$ & $103.0 \pm 8.6$ & $9.7 \pm 2.1$ & $780.2 \pm 146.3$ \\
\hline YM11 ( $\Delta h d f 1 / H D F 1)$ & $11.0 \pm 0.4$ & $102.0 \pm 7.9$ & $11.2 \pm 3.8$ & $890.8 \pm 201.5$ \\
\hline
\end{tabular}

\footnotetext{
${ }^{a}$ Mean and standard deviation were calculated from three independent replicates.

$\mathrm{b}$ Average daily extension in colony radius on potato dextrose agar plates.

${ }^{c}$ Conidiation in 5-day-old CMC cultures.

${ }^{\mathrm{d}}$ Diseased spikelets per wheat head.

e Deoxynivalenol.
} 
of vegetative hyphae or 18-h germlings (data not shown). Weak GFP signals were observed in the nucleus only in freshly harvested conidia (Fig. 8A).

The expression level of HDFl in conidia, germlings (12 h), and vegetative hyphae of the wild-type strain was quantified by quantitative reverse-transcription polymerase chain reaction (qRT-PCR). Transcripts of HDFl were abundant in conidia, reduced in 12-h germlings, and lowest in vegetative hyphae, which were 2.4-fold lower than those of conidia (Fig. 8B). These results indicate that $H D F 1$ had the highest expression level in conidia, which agreed with the observation that GFP signals were only observed in the nucleus of conidia in $H D F 1$ eGFP transformant YM9.

\section{Microarray analysis with the $\Delta h d f 1$ mutant.}

To identify genes regulated by $H D F 1$, the whole-genome GeneChip of $F$. graminearum (Guldener et al. 2006) was used for microarray analysis with RNA samples isolated from 18-h germlings. In comparison with the wild type, 149 and 253 genes were down- and upregulated, respectively, over fivefold in the $\Delta h d f 1$ mutant (Supplementary Tables 2 and 3). The largest MIPS (Munich Information Center for Protein Sequences) category (Mewes et al. 2002) of the downregulated genes $(43 \%, n=61)$ encodes proteins involved in metabolism (Supplementary Fig. 1). A number of genes involved in nitrogen metabolism, including the nitrate (FGSG_01947) and nitrite (FGSG_08402) reductase genes, were significantly reduced in the mutant. The second largest group of the downregulated genes $(29 \%, n=42)$ consists of hypothetical proteins with unknown biological functions.

A
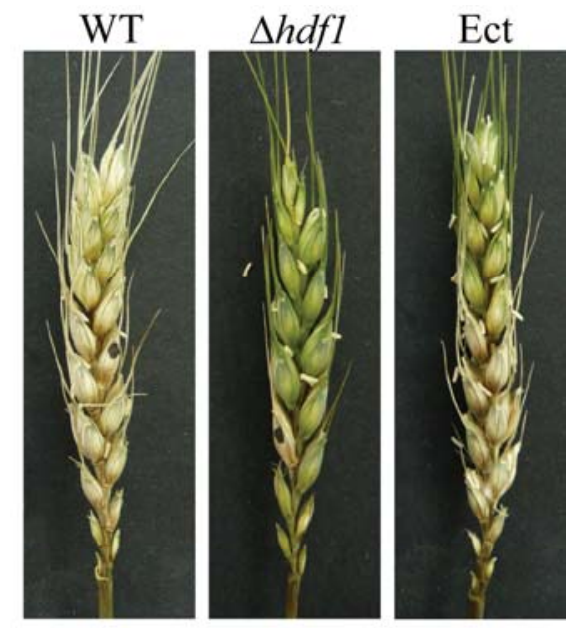

B

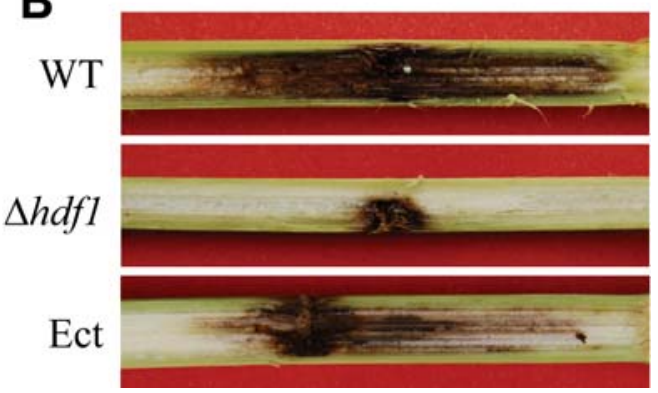

Fig. 3. Infection assays with flowering wheat heads and corn stalks. A, Flowering wheat heads were drop inoculated with conidia of the wild type (WT), $\Delta h d f 1$ mutant YM1, or an ectopic transformant (Ect). Typical wheat heads were examined 14 days postinoculation (dpi). B, Corn stalks inoculated with WT, $\Delta h d f 1$ mutant, or Ect were examined 14 dpi. The $\Delta h d f 1$ mutant failed to cause extensive discoloration.
The gene with the most significantly reduced expression level in the $\Delta h d f 1$ mutant was FGSG_03168 (downregulated 124-fold), which is predicted to encode a putative fructose transporter. Interestingly, in total, 22 transport-related genes belonging to MIPS category 20 (Mewes et al. 2002), including eight additional sugar transporter and four putative amino acid permease or transporter genes, were reduced more than fivefold in their expression levels, which constituted the third largest group of genes downregulated in the $\Delta h d f 1$ mutant. According to microarray data deposited in PlexDB, six of these transport-related genes-FGSG_00136 (9.1×), FGSG_04182 (3.4×), FGSG_04204 (2.0×), FGSG_05839 (6.1×), FGSG_ $07631(3.4 \times)$, and FGSG_11480 (3.1×) - have increased expression levels in infected barley heads at $144 \mathrm{hpi}$. Another six had increased expression levels during colonization of wheat stems (Guenther et al. 2009). Some of these transport-related genes may play a role in plant infection.

To verify the microarray data, eight genes were selected for qRT-PCR analysis. All of them were confirmed to be significantly downregulated in the $\Delta h d f 1$ mutant (Fig. 9A). FG03882 encodes a putative $\mathrm{ABC}$ transporter that is similar to $A B C 1$, a
A

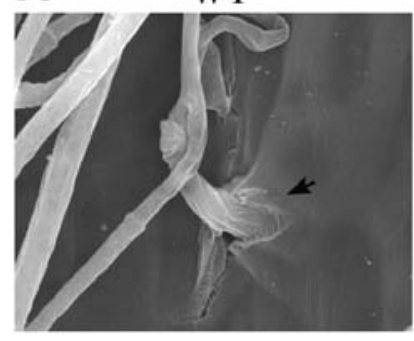

B
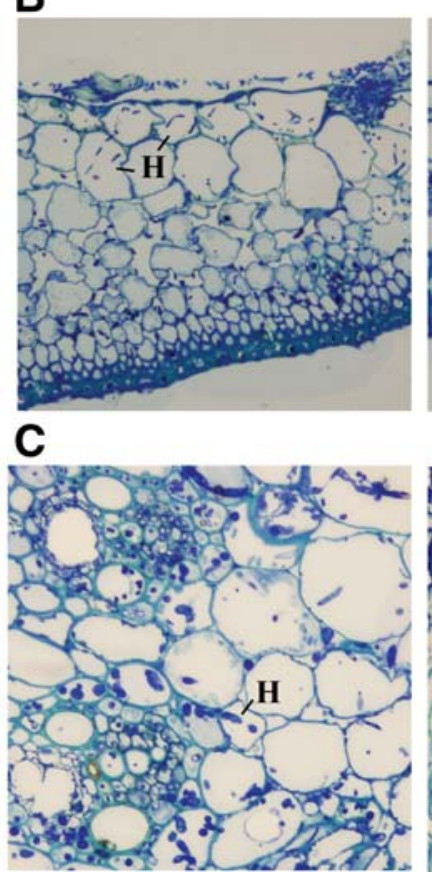
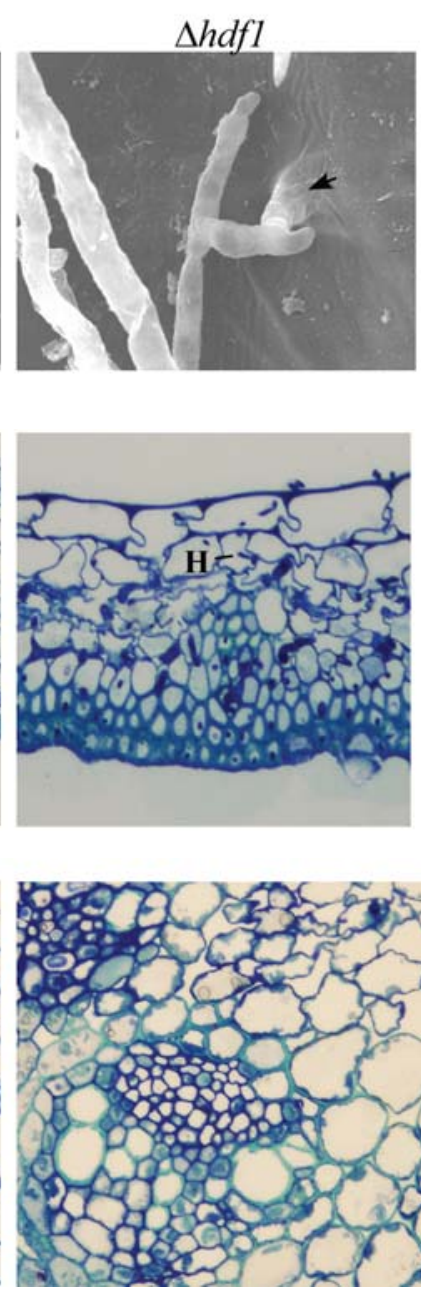

Fig. 4. Electron micrographs of penetration and colonization of flowering wheat heads. A, Lemma from the spikelets inoculated with the wild-type (WT) (PH-1) and $\Delta h d f 1$ mutant strains were examined by scanning electron microscopy $24 \mathrm{~h}$ postinoculation (hpi). Penetration sites on the inner lemma surface are marked with arrows. B, Colonization of lemma tissues by WT PH-1 and the $\Delta h d f 1$ mutant 48 hpi. C, Rachis directly beneath the inoculated spikelet was examined by transmission electron microscopy 120 hpi. In samples inoculated with the WT, abundant hyphae were observed. No hyphae were observed in the rachis below the spikelets inoculated with the $\Delta h d f 1$ mutant. $\mathrm{H}$, hyphae. 
transporter gene known to be important for pathogenesis in $M$. oryzae (Urban et al. 1999). Although the exact molecular mechanism is not clear, $A B C 1$ may play a critical role in the transportation or avoidance of plant defense compounds. FG03675 encodes the autophagy protein Atg22. In M. oryzae, autophagy plays an important role in appressorium penetration and plant infection (Kershaw and Talbot 2009). FG09921 encodes a fungal-specific transcription factor that is orthologous to a transcriptional regulator of acetate utilization in $A$. nidulans and Neurospora crassa (Bibbins. et al. 2002; Todd et al. 1997). The downregulation of some of these genes may contribute to reduced virulence of the $\Delta h d f 1$ mutant.

For the genes that are upregulated in the $\Delta h d f 1$ mutant, the top two categories were hypothetical proteins $(73 \%)$ and pro-
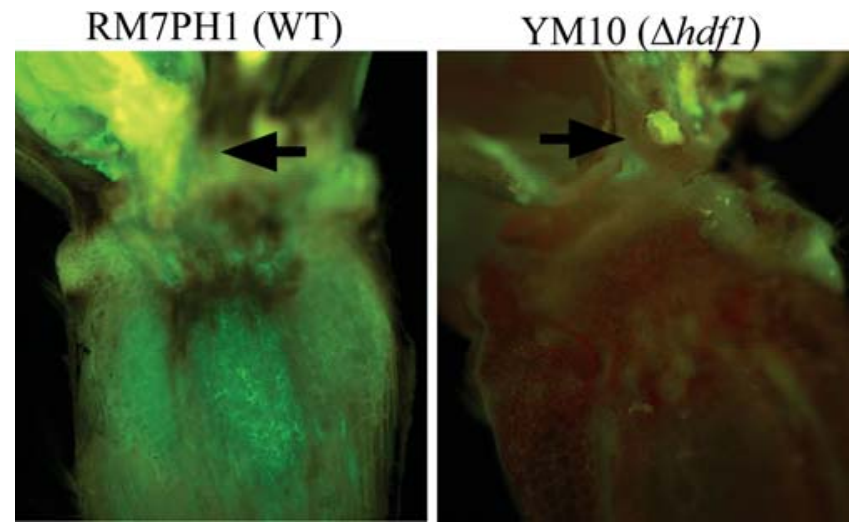

Fig. 5. Defects in spreading from inoculated spikelets to the rachis. Wheat heads inoculated with transformants of the wild type (WT) (RM7PH1) and the $\Delta h d f 1$ mutant (YM10) expressing the $\mathrm{P}_{\mathrm{RP} 27}$-eGFP construct were examined under an epifluorescence microscope 5 days postinoculation. Arrows mark the site where the inoculated spikelet was removed. Green fluorescent protein (GFP) signals representing fungal growth were visible in rachis tissues from wheat heads inoculated with transformant RM7PH1 but not YM10.

A

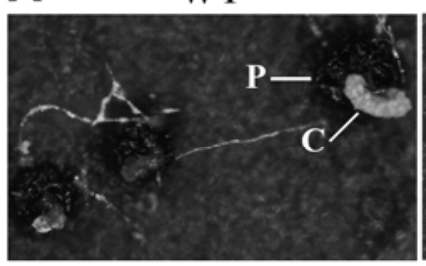

B

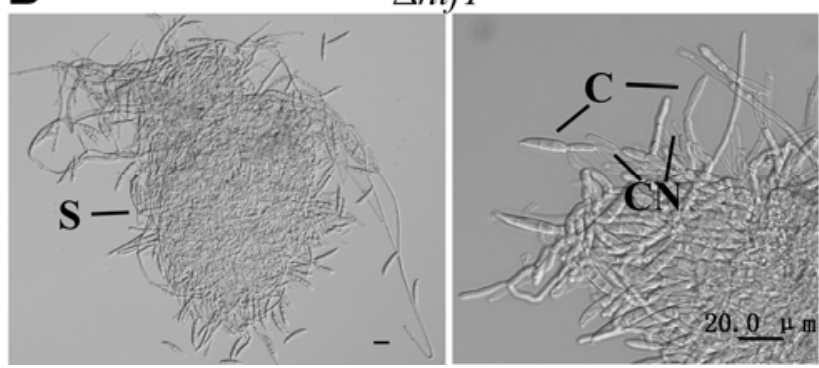

Fig. 6. $\Delta h d f 1$ mutant is female sterile but produces abundant sporodochia. A, Carrot agar cultures of the wild type (WT) and $\Delta h d f 1$ mutant (YM1) were photographed 15 days after self-fertilization. Arrows mark mature perithecia with ascospore cirrhi. Abundant sporodochia are visible as tufts (marked with arrows) on the colony surface of the $\Delta h d f 1$ mutant. P, perithecia; S, sporodochia. B, Conidiophores and macroconidia (right panel) produced by the $\Delta h d f 1$ mutant in sporodochia/tufts (left panel) formed on carrot agar plates. Bar $=20 \mu \mathrm{m}$. C, conidia; $\mathrm{CN}$, conidiophores. teins involved in metabolism (18\%). Almost $75 \%$ of the genes upregulated in the $\Delta h d f 1$ mutant encode proteins with unknown functions. Many of them are unique to filamentous fungi or Fusarium spp. Interestingly, six members of the autofusarin biosynthesis gene cluster (FGSG_2324 to FGSG_ 2329) were upregulated over 15 -fold in the $\Delta h d f 1$ mutant (Supplementary Table 4). Eight of the upregulated genes, including FGSG_06596 (putative catalase) and FGSG_08151 (putative peroxidase), were selected for qRT-PCR analysis (Fig. 9B). All but FGSG_07798 were confirmed to be signifycantly upregulated in the $\Delta h d f 1$ mutant (Fig. 9B).

Because of the increased expression of FGSG_06596 and FGSG_08151 in the $\Delta h d f 1$ mutant, we assayed hyphal growth in the presence of different concentrations of $\mathrm{H}_{2} \mathrm{O}_{2}$. On PDA

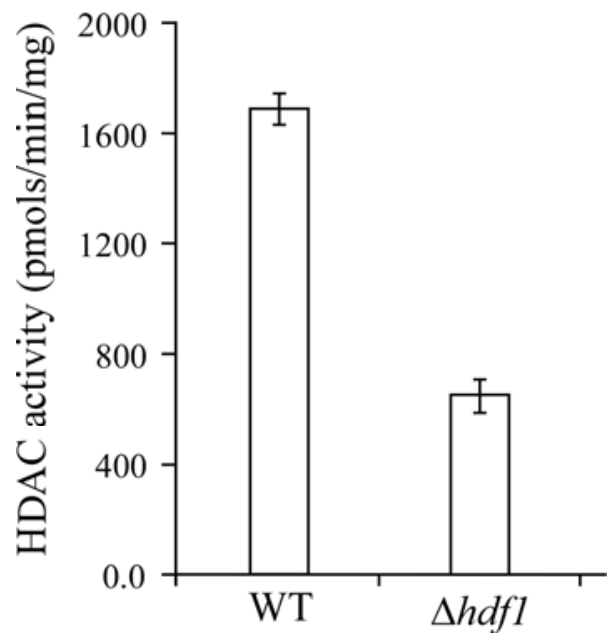

Fig. 7. Assays for histone deacetylase (HDAC) activities. Protein extracts of the wild type and $\Delta h d f 1$ mutant YM1 were assayed for HDAC activities with the colorimetric HDAC assay kit from (Active Motif). The $\Delta h d f 1$ mutant is reduced approximately $60 \%$ in HDAC activity.
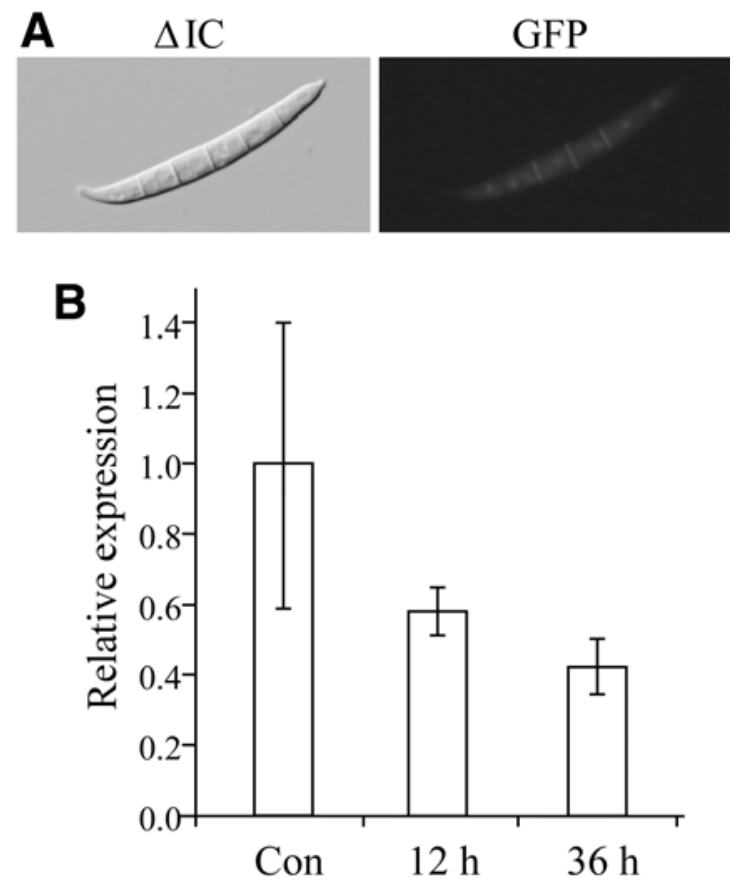

Fig. 8. Expression and localization of $H D F 1$. A, Nuclear localization of Hdf1-eGFP fusion proteins in conidia of transformant YM9. B, Relative expression level of $H D F 1$ in conidia, 12-h germlings, and 36-h vegetative hyphae of the wild type was analyzed with the $2^{-\Delta \Delta \mathrm{CT}}$ method. Expression level in conidia was arbitrarily set to 1 . GFP = green fluorescent protein. 
with $0.05 \% \mathrm{H}_{2} \mathrm{O}_{2}$, vegetative growth was inhibited in the wild type but the $\Delta h d f 1$ mutant formed colonies with limited aerial hyphae (Fig. 9C). Under the same conditions, the complemented transformant, similar to the wild type, was completely inhibited in aerial hyphal growth (Fig. 9C). These data indicate that the $\Delta h d f 1$ mutant was more tolerant to $\mathrm{H}_{2} \mathrm{O}_{2}$ than the wild type.

\section{Functional characterization}

of the HDA1 and $\mathrm{HOS} 3$ orthologs in $\mathrm{F}$. graminearum.

To determine the functions of the other two class II HDAC genes that are homologous to yeast HDAl and HOS3 (designated $H D F 2$ and $H D F 3$ in this study), we used the split-marker approach to generate the $\Delta h d f 2$ and $\Delta h d f 3$ mutants. Unlike the $H D F 1$ gene, $H D F 2$ and $H D F 3$ appeared to be dispensable for vegetative growth. The $\Delta h d f 2$ and $\Delta h d f 3$ mutants had a normal growth rate (Supplementary Fig. 2). The $\Delta h d f 2$ mutant was

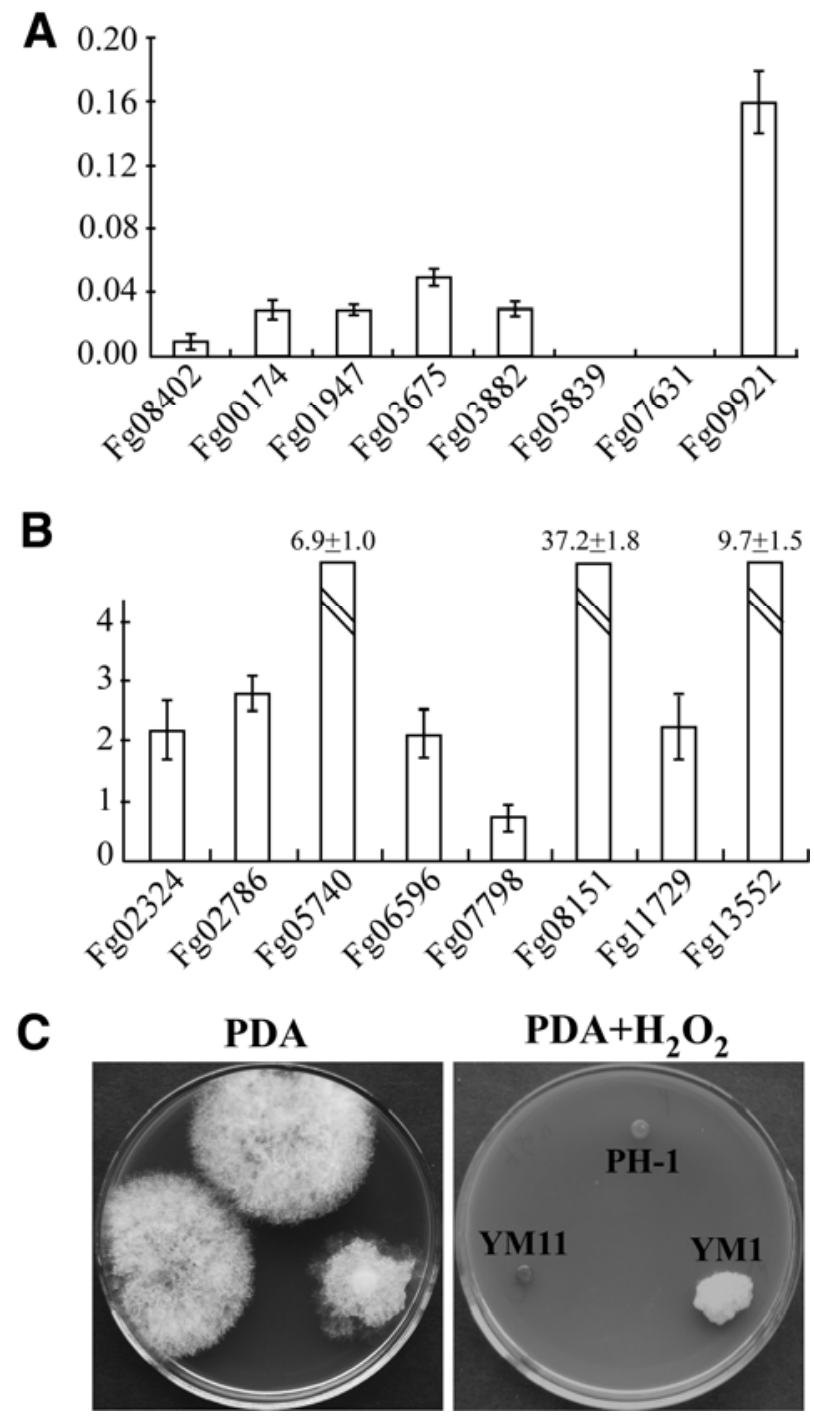

Fig. 9. Verification of selected genes from microarray analysis with the $\Delta h d f 1$ mutant. RNA samples isolated from 18-h germlings of the wild type and $\Delta h d f 1$ mutant were used for quantitative reverse-transcription polymerase chain reaction analysis. Relative expression level of individual genes in the wild type was arbitrarily set to 1 . Prefix of gene numbers is abbreviated from FGSG $_{-}$to Fg. A, Relative expression levels of eight genes downregulated in the $\Delta h d f 1$ mutant. $\mathbf{B}$, Relative expression levels of eight genes with increased expression levels in the microarray data of the $\Delta h d f 1$ mutant. C, Colonies of the wild-type (PH-1), complemented transformant YM11 ( $\Delta h d f 1 / H D F 1)$, and $\Delta h d f 1$ mutant YM1 formed on potato dextrose agar (PDA) plates with $0.05 \% \mathrm{H}_{2} \mathrm{O}_{2}$. slightly reduced in conidiation and virulence in infection assays with flowering wheat heads (Table 2; Fig. 10A). In contrast, the $\Delta h d f 3$ mutant had no significant change in conidiation and virulence (Table 2; Fig. 10A).

To determine whether these two class II HDAC genes have overlapping functions with $H D F 1$, we generated the $\Delta h d f 1$ $\Delta h d f 2$ and $\Delta h d f 1 \Delta h d f 3$ double mutants. Deletion of either $H D F 2$ or $H D F 3$ in the $\triangle h d f 1$ mutant resulted in a more severe reduction in growth rate. On PDA plates, the growth rates of the $\Delta h d f 1 \Delta h d f 2$ and $\Delta h d f 1 \Delta h d f 3$ mutants were $6.9 \pm 2.0$ and $7.9 \pm 1.0 \mathrm{~mm} /$ day, respectively, which were slower than that of the wild type and $\Delta h d f 1$ mutant (Table 2). In wheat head infection assays, the $\Delta h d f 1 \Delta h d f 2$ and $\Delta h d f 1 \Delta h d f 3$ mutants were more significantly reduced in virulence (Fig. 10A). Typical head blight symptoms were observed in only approximately $50 \%$ of the inoculated spikelets. No spreading from the inoculated spikelets to other wheat kernels was observed in these two double mutants. Similar results were observed in infection assays with corn stalks (Fig. 10B). At 14 dpi, only limited discoloration was observed in the corn stalks at the inoculation sites inoculated with the $\Delta h d f 1 \Delta h d f 2$ and $\Delta h d f 1 \Delta h d f 3 \mathrm{mu}-$ tants. These results indicate that both $H D F 2$ and $H D F 3$ have a minor role in the regulation of vegetative growth and plant infection in $F$. graminearum.

\section{DISCUSSION}

The reversible acetylation and deacetylation of lysine residues in the tail of core histones are common forms of histone modifications mediated by HAT and HDAC, respectively. Whereas HAT act as transcriptional coactivators, HDAC function as transcriptional corepressors. HDAC genes are divided into three classes (Yang and Grégoire 2005). Class I and class II include the classical HDAC that are sensitive to inhibition

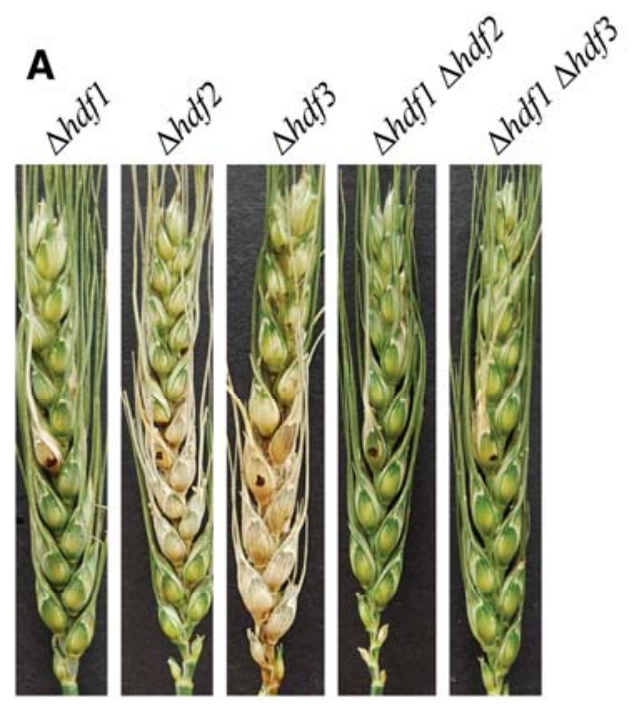

B

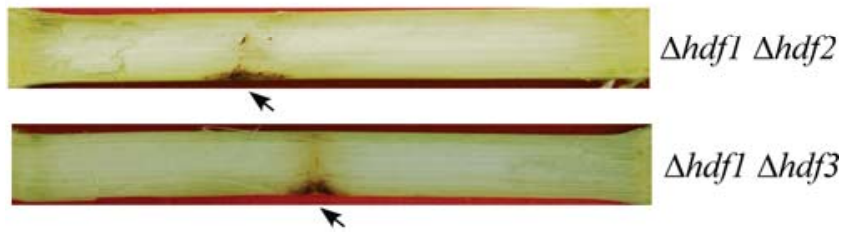

Fig. 10. Infection assays with flowering wheat heads and corn stalks. A, Wheat heads inoculated with conidia from the $\Delta h d f 1, \Delta h d f 2, \Delta h d f 3, \Delta h d f 1$ $\Delta h d f 2$, and $\Delta h d f 1 \Delta h d f 3$ mutants were photographed 14 days postinoculation (dpi). B, Corn stalks inoculated with the $\Delta h d f 1 \Delta h d f 2$ and $\Delta h d f 1$ $\Delta h d f 3$ double mutants were examined 14 dpi. 
by trichostatin A and share similarity to yeast Rpd3 and Hda1, respectively. In addition to three class II HDAC genes characterized in this study, the $F$. graminearum genome has one predicted class I HDAC gene (FGSG_00870) that is highly homologous to yeast RPD3. Phylogenetic analysis indicates that these four class I and class II HDAC genes are well conserved in filamentous fungi and can be divided into four clades represented by yeast RPD3, HOS2, HDA 1, and HOS3.

The Hdf1 HDAC is orthologous to yeast Hos2, which interacts with Sif2 as components of the Set3 complex (Cohen et al. 2008). In yeast two-hybrid assays, Hdf1 interacted with Ftl1, an ortholog of Sif2 in F. graminearum (Ding et al. 2009). Deletion of $H D F 1$ significantly reduced the virulence in infection assays with flowering wheat heads and corn stalks. Similar to the $\Delta f t l l$ mutant (Ding et al. 2009), the $\Delta h d f l$ mutant was defective in spreading from colonized spikelets into the rachis. In $C$. carbonum, the $h d c l$ mutant was significantly compromised in colonizing corn leaf tissues (Baidyaroy et al. 2001). In M. oryzae, the core components of the Tig1 HDAC complex, including orthologs of yeast Hos2, Sif2, Snt1, and Set3, all are important for plant infection (Ding et al. 2010). Preliminary analysis with mutants deleted of the $F g S N T 1$ gene indicated that the SNT1 ortholog also was important for pathogenesis in F. graminearum (S. Ding and J.-R. Xu, unpublished). These observations suggest that Hdf1 and Ftl1 are components of a well-conserved HDAC complex in the wheat scab fungus for regulating plant infection processes.

One common stress faced by hyphae of necrotrophic fungi in planta is reactive oxygen species (ROS) generated during oxidative burst. The $\Delta f t l l$ mutant had increased sensitivity to $\mathrm{H}_{2} \mathrm{O}_{2}$, which may contribute to its defects in plant infection (Ding et al. 2009). To our surprise, the $\Delta h d f 1$ mutant had increased tolerance to $0.05 \% \mathrm{H}_{2} \mathrm{O}_{2}$ and upregulated expression of putative catalase and peroxidase genes, suggesting that FTL1 and HDF1 differ in subsets of genes that they regulate in $F$. graminearum. We also assayed responses of the $\Delta h d f 1 \mathrm{mu}-$ tant to stresses. Although it had no obvious defects in growth or conidium germination in the presence of $0.7 \mathrm{M} \mathrm{NaCl}$ or $1 \mathrm{M}$ sorbitol (data not shown), the mutant was more sensitive to $0.01 \%$ sodium dodecyl sulfate (SDS) than the wild-type strain (Supplementary Fig. 3A), suggesting a defect in the cytoplasm membrane. Five genes involved in ergosterol synthesis (FGSG_01836, FGSG_03686, FGSG_05740, FGSG_09031, and FGSG_11044) were upregulated in the $\Delta h d f 1$ mutant, which is indicative of membrane stress. The $\Delta h d f 1$ mutant also had increased sensitivity to cell wall stresses and cell-wall-degrading enzymes, suggesting that the $\Delta h d f l$ mutant was defective in cell wall integrity. In addition, the $\Delta h d f 1$ mutant was reduced in the production of DON, which is one of the bestcharacterized virulence factors in $F$. graminearum (Maier et al. 2006; Proctor et al. 1997). It also had a defect in vegetative growth (reduced growth rate). All of these defects of the $\Delta h d f 1$ mutant may contribute to its reduced virulence.

The $\Delta h d f 1$ mutant was defective in sexual reproduction. On mating plates, instead of forming perithecia or protoperithecia, the $\Delta h d f 1$ mutant produced abundant sporodochia and macroconidia (Fig. 6). Although it was reduced in conidiation in liquid $\mathrm{CMC}$ cultures, asexual reproduction appeared to be stimulated in the $\Delta h d f l$ mutant on carrot agar plates under culture conditions conducive for sexual reproduction. It is possible that $F g H O S 2$ plays a role in suppressing the production of sporodochia and conidia when sexual reproduction is activated on mating plates. In liquid cultures, $F$. graminearum does not produce sporodochia and conidia are formed on individual conidiophores. The difference between liquid CMC and mating cultures could be related to the regulation of sporodochium formation by $\mathrm{FgHOS} 2$. In M. oryzae, deletion of $\mathrm{MoHOS} 2$ also results in sexual reproduction defects (Ding et al. 2010) but conidiation was not stimulated in mating cultures of the Mohos 2 mutant (data not shown). The difference between the $\Delta h d f 1$ and Mohos 2 mutants in conidiation on mating plates may be related to different mating systems in $F$. graminearum (homothallic) and $M$. oryzae (heterothallic).

Unlike the Mghos2 mutant of M. oryzae that formed abnormal conidia (Ding et al. 2010), the $\Delta h d f 1$ mutant had no obvious defects in conidium morphology (Supplementary Fig. 4). The $\Delta h d f 2$ and $\Delta h d f 3$ mutants also produced normal conidia. However, majority of the conidia produced by the $\Delta h d f 1 \Delta h d f 2$ double mutant were shorter and wider than the wild-type conidia. In the $\Delta h d f 1 \Delta h d f 3$ mutant, most of the conidia had similar defects in morphology, although to a lesser extent. Therefore, $H D F 2$ and $H D F 3$ may have overlapping functions with $H D F 1$ during conidiogenesis or conidium development.

Microarray analysis revealed that more genes were upregulated than downregulated (253 versus 149) in the $\Delta h d f 1 \mathrm{mu}-$ tant. This is not surprising because HDAC usually function as transcriptional corepressors. However, it is unexpected that the expression of autofusarin biosynthesis genes was significantly increased because the $\Delta h d f 1$ mutant formed whitish colonies on PDA plates (Fig. 2C). FGSG_03064 and FGSG_03065, orthologs of the $A L-1$ and $A L-2$ genes involved in carotenoid biosynthesis (Jin et al. 2010; Li and Schmidhauser 1995), also were upregulated over 15-fold in the mutant. The discrepancy between colony pigmentation and microarray data could be related to culture conditions. RNA samples for microarray experiments were isolated from 18-h germlings grown in liquid CM. In fact, pigmentation in liquid cultures of the $\Delta h d f 1$ mutant tended to be stronger than that of the wild type (Supplementary Fig. 5). In A. fumigatus, deletion of the HOS2 ortholog has not been reported but deletion of the hdaA HDAC gene results in the upregulation of secondary metabolism (Lee et al. 2009).

In comparison with $H D F 1$, the other two class II HDAC genes appeared to be less important in $F$. graminearum. The $\Delta h d f 2$ and $\Delta h d f 3$ mutants were normal in growth rate. The $\Delta h d f 3$ mutant was normal and the $\Delta h d f 2$ mutant was only slightly reduced in conidiation and virulence. Significant reduction in conidiation and virulence was observed only in the $\Delta h d f 1$ mutant, which had a $60 \%$ reduction in HDAC activity (Fig. 7). These results indicate that $H D F 1$ is the major class II HDAC gene in $F$. graminearum and it plays a more important role than $H D F 2$ and $H D F 3$. In A. nidulans, the HdaA (ortholog of hdf2) was reported to be the major HDAC gene (Tribus et al. 2005), which is contradictory to the recent finding of RpdA being an essential HDAC gene (Tribus et al. 2010). Because the $\Delta h d f 1 \Delta h d f 2$ and $\Delta h d f 1 \Delta h d f 3$ double mutants displayed more severe defects in growth and plant infection than the $\Delta h d f 1$ mutant, these three HDAC genes must have both overlapping and distinct functions. Therefore, it will be interesting to compare the expression profiles of these HDAC mutants. Comparative analysis will help to determine subsets of genes regulated by different class II HDAC genes and their interactions in regulating genes important for growth, conidiation, and pathogenesis.

\section{MATERIALS AND METHODS}

Strains and culture conditions.

The wild-type strain and mutants of $F$. graminearum used in this study are listed in Table 1 . Cultures were routinely grown on PDA plates at $25^{\circ} \mathrm{C}$ (Hou et al. 2002; Seong et al. 2006). Conidiation in 5-day-old CMC cultures and growth rate on PDA plates were measured as described (Ding et al. 2009; Zhou et al. 2010). For DNA, RNA, and protein extraction, 
vegetative hyphae were harvested from liquid YEPD (1\% yeast extract, $2 \%$ peptone, $2 \%$ glucose) cultures. Protoplast preparation and fungal transformation were performed as described (Hou et al. 2002; Proctor et al. 1995). For transformation, hygromycin B (Calbiochem, La Jolla, CA, U.S.A.) and geneticin (Sigma-Aldrich, St. Louis) were added to the final concentration of 250 and $150 \mu \mathrm{g} / \mathrm{ml}$, respectively. For testing sensitivities to various stresses, fungal growth was assayed after incubation at $25^{\circ} \mathrm{C}$ for 3 days on $\mathrm{CM}$ plates with $1 \mathrm{M}$ sorbitol, $0.7 \% \mathrm{NaCl}$ (wt/vol), $0.01 \%$ SDS (wt/vol), or different concentrations of $\mathrm{H}_{2} \mathrm{O}_{2}$ (Liu et al. 2011).

\section{Plant infection assays.}

For wheat head and corn stalk infection assays, freshly harvested conidia were resuspended to $10^{6} \mathrm{spores} / \mathrm{ml}$ in sterile water. Flowering wheat heads of cultivars Norm and Xiaoyan22 were inoculated with $10 \mu \mathrm{l}$ of conidium suspensions at the fifth spikelet from the base of the spike (Gale et al. 2002; Kang and Buchenauer 1999). Spikelets with typical head blight symptoms were counted 14 dpi. Diseased kernels were collected and assayed for DON and ergosterol production as described (Bluhm et al. 2007; Seong et al. 2006). Stalks of 8-week-old corn plants of cv. Pioneer 2375 were inoculated with toothpicks dipped in conidium suspensions as described (Choi and Xu 2010; Zhou et al. 2010). Stalk rot symptoms were examined after splitting the stalks longitudinally along the inoculation site 14 dpi.

\section{Generation of the $\Delta h d f 1, \Delta h d f 2$, and $\Delta h d f 3$ mutants.}

The split-marker approach was used to generate the HDF1 gene replacement mutant. The $0.54-\mathrm{kb}$ upstream and $0.55-\mathrm{kb}$ downstream flanking sequences of the $H D F 1$ gene were amplified with primer pairs Hdf1F1-Hdf1R2 and Hdf1F3-Hdf1R4 and fused with fragments of the $h p h$ gene amplified with primers HY/R, YG/F, HYG/F, and HYG/R by overlapping PCR as described (Catlett et al. 2003; Zhou et al. 2010). The resulting PCR products were transformed into protoplasts of the wildtype strain PH-1. Putative $\Delta h d f 1$ mutants were identified by PCR with primer pairs Hdf1F5-df1R6, Hdf1F7-H855R, H856F-df1R8, and H852-H850 and confirmed by Southern blot hybridization. The same split-marker approach was used to generate the $\Delta h d f 2$ and $\Delta h d f 3$ mutants.

\section{Generation of the $\Delta h d f 1 \Delta h d f 2$ and $\Delta h d f 1 \Delta h d f 3$ double mutants.}

To generate the double mutants, the geneticin-resistance $\left(n e o^{\mathrm{R}}\right)$ gene was used as the selectable marker to delete the $H D F 1$ gene in the $\Delta h d f 2$ or $\Delta h d f 3$ mutant. Primer pairs GEN/F-GE/R and EN/F-GEN/R were used to amplify the split-marker fragments of the $n e o^{\mathrm{R}}$ gene from plasmid $\mathrm{pFL} 2$, which was generated by replacing the $h p h$ gene on pDL2 with the geneticin-resistance marker (Bourett et al. 2002; Bruno et al. 2004). Primers pairs dm $1 f-d m 2 r$ and dm $3 f-d m 4 r$ were used to amplify the $0.83-\mathrm{kb}$ upstream and $0.79-\mathrm{kb}$ downstream flanking sequences of $H D F 1$, respectively. The $n e o^{\mathrm{R}}$ fragment amplified with primers N850 and N852 was used as the probe for Southern blot analysis.

\section{Complementation of the $\Delta h d f 1$ mutant with $H D F 1$ and $H D F 1$-eGFP fusion.}

For complementation assays, a 3.4-kb fragment of the $H D F 1$ gene (containing the $1.5-\mathrm{kb}$ promoter region) was amplified with primers Gloc-h1F and Gloc-h1R and cloned into pFL2 by the gap repair method (Bourett et al. 2002; Bruno et al. 2004). The resulting complementation construct was transformed into the $\Delta h d f 1$ mutant YM1. The HDF1-eGFP construct was generated by cloning the $H D F 1$ fragment amplified with primers Gloc-h1F and Gloc-h1R into pKB04 (Bruno et al. 2004). After transforming into protoplasts of YM1, transformants expressing the $H D F 1$ complementation and $H D F 1$ eGFP constructs were analyzed by PCR. GFP signals were observed under an Olympus BX51 epifluorescence microscope.

\section{qRT-PCR analysis.}

RNA samples were isolated from conidia, germlings, and mycelia with the TRIzol reagent (Invitrogen, Carlsbad, CA, U.S.A.). First-strand cDNA was synthesized with the Fermentas 1st cDNA synthesis kit (Hanover, MD) following the instructtions provided by the manufacturer. Primers used for qRT-PCR analysis are listed in Supplementary Table 1 . The $F$. graminearum $\beta$-tubulin (TUB2) gene was amplified with primers TubQF and TubQR (Bluhm et al. 2007). Relative changes in the expression level of each gene were calculated by the $2^{-\Delta \Delta C t}$ method (Livak and Schmittgen 2001) with TUB2 as the endogenous reference. For each gene, qRT-PCR data from three biological replicates were used to calculate the mean and standard deviation.

\section{SEM and TEM observations.}

Glumes and lemmas were collected from inoculated spikelets and fixed with $4 \%$ ( $\mathrm{vol} / \mathrm{vol})$ glutaraldehyde in $0.1 \mathrm{M}$ phosphate buffer ( $\mathrm{pH} 6.8$ ) overnight at $4^{\circ} \mathrm{C}$ and rinsed with the same buffer for $2 \mathrm{~h}$. After dehydration in a graded series of acetone $(30,50,70,80,90$, and $100 \%$; vol/vol), the samples were mounted on stubs, sputter coated with gold-palladium, and examined with a JEOL 6360 scanning microscope (Jeol Ltd., Tokyo). For TEM examination, slices of wheat glumes and rachis tissues were fixed, dehydrated, and embedded as described (Ding et al. 2009). Ultrathin sections were treated with uranyl acetate and lead citrate (Kang et al. 2008) and examined with a JEM-1230 electron microscope (Jeol Ltd.) at $80 \mathrm{KV}$. At least three independent biological replicates were examined for the wild-type and $\Delta h d f 1$ mutant strains.

\section{Microarray analysis.}

Freshly harvested conidia of PH-1 and YM1 (Table 1) were resuspended to $10^{6}$ conidia/ml in $100 \mathrm{ml}$ of CM. After incubation at $25^{\circ} \mathrm{C}$ for $18 \mathrm{~h}$, germinated conidia were collected by filtration and used for RNA isolation with the TRIzol reagent (Invitrogen). For each strain, RNA was isolated from three biological replicates. Probe labeling and hybridization of the Fusarium GeneChip microarrays (Guldener et al. 2006) were performed with standard Affymetrix procedures at the Purdue Core Genomics Facility. Hybridization signals were scanned with a GeneChip GCS 3000 scanner (Affymetrix, Santa Clara, CA, U.S.A.). The resulting CEL files were processed with the Affymetrix MAS5.0 system and analyzed with GeneSpring GX V7.2 (Agilent Technologies, Santa Clara, CA, U.S.A.).

\section{HDAC activity assays.}

Vegetative hyphae of the wild-type PH-1 and the $\Delta h d f 1 \mathrm{mu}-$ tant were harvested from $100 \mathrm{ml}$ of 3-day-old CM cultures and resuspended in $400 \mu \mathrm{l}$ of lysis buffer (Ding et al. 2009) with 5 $\mu \mathrm{l}$ of protease inhibitor cocktail (Sigma-Aldrich). After homogenization with acid-washed glass beads in a Biospec mini-bead beater for three 40-s pulses with 1-min intervals on ice, the lysate was separated from the glass beads and centrifuged at $16,000 \times g$ for $15 \mathrm{~min}$ at $4^{\circ} \mathrm{C}$ (Ding et al. 2009). The resulting supernatants were used to assay for HDAC activities with the colorimetric HDAC assay kit (Active Motif, Carlsbad, CA, U.S.A.) following the instructions provided by the manufacturer. Absorbance was detected with a plate reader (Synergy HT, Bio-TEK, Houston, TX, U.S.A.; 360-nm excitation and 465-nm emission). The concentration of deacetylated com- 
pounds was calculated from the deacetylation standard curve and used to estimate the HDAC activity (pmoles per minute per milligram) with the formula provided with the Active Motif HDAC assay kit.

\section{ACKNOWLEDGMENTS}

We thank L. Dunkle and S. Goodwin at Purdue University for critical reading of this manuscript, Q. Hang and K. Zhang at Northwestern A\&F University for technical assistance during this study, and Y. Dong for assistance with DON detection. This work was supported by the 111 Project from the Ministry of Education of China (B07049) and grants to J.-R. Xu from the National Research Initiative of the United States Department of Agriculture Cooperative State Research, Education and Extension Service (no. 2007-35319-102681) and the US WBSI.

\section{LITERATURE CITED}

Bai, G. H., and Shaner, G. 2004. Management and resistance in wheat and barley to Fusarium head blight. Annu. Rev. Phytopathol. 42:135-161.

Baidyaroy, D., Brosch, G., Ahn, J. H., Graessle, S., Wegener, S., Tonukari, N. J., Caballero, O., Loidl, P., and Walton, J. D. 2001. A gene related to yeast HOS2 histone deacetylase affects extracellular depolymerase expression and virulence in a plant pathogenic fungus. Plant Cell 13:1609-1624.

Bibbins, M., Crepin, V. F., Cummings, N. J., Mizote, T., Baker, K., Mellits, K. H., and Connerton, I. F. 2002. A regulator gene for acetate utilisation from Neurospora crassa. Mol. Genet. Genomics 267:498-505.

Bluhm, B. H., Zhao, X., Flaherty, J. E., Xu, J. R., and Dunkle, L. D. 2007. RAS2 regulates growth and pathogenesis in Fusarium graminearum. Mol. Plant-Microbe Interact. 20:627-636.

Bourett, T. M., Sweigard, J. A., Czymmek, K. J., Carroll, A., and Howard, R. J. 2002. Reef coral fluorescent proteins for visualizing fungal pathogens. Fungal Genet. Biol. 37:211-220.

Brown, N. A., Urban, M., Van De Meene, A. M. L., and HammondKosack, K. E. 2010. The infection biology of Fusarium graminearum: Defining the pathways of spikelet to spikelet colonisation in wheat ears. Fungal Biol. 114:555-571.

Bruno, K. S., Tenjo, F., Li, L., Hamer, J. E., and Xu, J. R. 2004. Cellular localization and role of kinase activity of $P M K 1$ in Magnaporthe grisea. Eukaryot. Cell 3:1525-1532.

Catlett, N. L., Lee, B., Yoder, O. C., and Turgeon, B. G. 2003. Split-marker recombination for efficient targeted deletion of fungal genes. Fungal Genet. Newsl. 50:9-11.

Choi, Y. E., and Xu, J.-R. 2010. The cAMP signaling pathway in Fusarium verticillioides is important for conidiation, plant infection, and stress responses but not fumonisin production. Mol. Plant-Microbe Interact. 23:522-533.

Cohen, T. J., Mallory, M. J., Strich, R., and Yao, T. P. 2008. Hos2p/Set3p deacetylase complex signals secretory stress through the Mpk1p cell integrity pathway. Eukaryot. Cell 7:1191-1199.

Cuomo, C. A., Gueldener, U., Xu, J. R., Trail, F., Turgeon, B. G., Di Pietro, A., Walton, J. D., Ma, L. J., Baker, S. E., Rep, M., Adam, G., Antoniw, J., Baldwin, T., Calvo, S., Chang, Y. L., DeCaprio, D., Gale, L. R., Gnerre, S., Goswami, R. S., Hammond-Kosack, K., Harris, L. J., Hilburn, K., Kennell, J. C., Kroken, S., Magnuson, J. K., Mannhaupt, G., Mauceli, E., Mewes, H. W., Mitterbauer, R., Muehlbauer, G., Munsterkotter, M., Nelson, D., O’Donnell, K., Ouellet, T., Qi, W. H., Quesneville, H., Roncero, M. I. G., Seong, K. Y., Tetko, I. V., Urban, M., Waalwijk, C., Ward, T. J., Yao, J. Q., Birren, B. W., and Kistler, H. C. 2007. The Fusarium graminearum genome reveals a link between localized polymorphism and pathogen specialization. Science 317:1400-1402.

Desjardins, A. E. 2003. Gibberella from A (venaceae) to Z (eae). Annu. Rev. Phytopathol. 41:177-198.

Ding, S., Liu, W., Liuk, A., Ribot, C., Vallet, J., Tao, A., Wang, Y., Lebrun, $\mathrm{M}$., and $\mathrm{Xu}, \mathrm{J}$. R. The Tig1 HDAC complex regulates infectious growth in the rice blast fungus Magnaporthe oryzae. Plant Cell. In press.

Ding, S. L., Mehrabi, R., Koten, C., Kang, Z. S., Wei, Y. D., Seong, K. Y., Kistler, H. C., and Xu, J. R. 2009. Transducin beta-like gene FTL1 is essential for pathogenesis in Fusarium graminearum. Eukaryot. Cell 8:867-876.

Gale, L. R., Chen, L. F., Hernick, C. A., Takamura, K., and Kistler, H. C. 2002. Population analysis of Fusarium graminearum from wheat fields in eastern China. Phytopathology 92:1315-1322.

Goswami, R. S., and Kistler, H. C. 2004. Heading for disaster: Fusarium graminearum on cereal crops. Mol. Plant Pathol. 5:515-525.

Graessle, S., Dangl, M., Haas, H., Mair, K., Trojer, P., Brandtner, E. M.,
Walton, J. D., Loidl, P., and Brosch, G. 2000. Characterization of two putative histone deacetylase genes from Aspergillus nidulans. Biochim. Biophys. Acta 1492:120-126.

Guenther, J. C., Hallen-Adams, H. E., Bucking, H., Shachar-Hill, Y., and Trail, F. 2009. Triacylglyceride metabolism by Fusarium graminearum during colonization and sexual development on wheat. Mol. PlantMicrobe Interact. 22:1492-1503.

Guldener, U., Seong, K. Y., Boddu, J., Cho, S. H., Trail, F., Xu, J. R., Adam, G., Mewes, H. W., Muehlbauer, G. J., and Kistler, H. C. 2006. Development of a Fusarium graminearum Affymetrix GeneChip for profiling fungal gene expression in vitro and in planta. Fungal Genet. Biol. 43:316-325.

Hou, Z. M., Xue, C. Y., Peng, Y. L., Katan, T., Kistler, H. C., and Xu, J. R. 2002. A mitogen-activated protein kinase gene (MGV1) in Fusarium graminearum is required for female fertility, heterokaryon formation, and plant infection. Mol. Plant-Microbe Interact. 15:1119-1127.

Jin, J. M., Lee, J., and Lee, Y. W. 2010. Characterization of carotenoid biosynthetic genes in the ascomycete Gibberella zeae. FEMS (Fed. Eur. Microbiol. Soc.) Microbiol. Lett. 302:197-202.

Kang, Z., and Buchenauer, H. 1999. Immunocytochemical localization of Fusarium toxins in infected wheat spikes by Fusarium culmorum. Physiol. Mol. Plant Pathol. 55:275-288.

Kang, Z. S., Buchenauer, H., Huang, L. L., Han, Q. M., and Zhang, H. C. 2008. Cytological and immunocytochemical studies on responses of wheat spikes of the resistant Chinese cv. Sumai 3 and the susceptible cv. Xiaoyan 22 to infection by Fusarium graminearum. Eur. J. Plant Pathol. 120:383-396.

Kershaw, M. J., and Talbot, N. J. 2009. Genome-wide functional analysis reveals that infection-associated fungal autophagy is necessary for rice blast disease. Proc. Natl. Acad. Sci. U.S.A. 106:15967-15972.

Lee, I., Oh, J., Shwab, E. K., Dagenais, T. R. T., Andes, D., and Keller, N. P. 2009. HdaA, a class 2 histone deacetylase of Aspergillus fumigatus, affects germination and secondary metabolite production. Fungal Genet. Biol. 46:782-790.

Li, C. G., and Schmidhauser, T. J. 1995. Developmental and photoregulation of al-1 and al-2, structural genes for 2 enzymes essential for carotenoid biosynthesis in Neurospora. Dev. Biol. 169:90-95.

Liu, W., Zhou, X., Li, G., Li, L., Kong, L., Wang, C., and Xu, J.-R. 2011. Multiple plant surface signals are sensed by different mechanisms in the rice blast fungus. PLoS Pathog. 7:e1001261. Published online.

Livak, K. J., and Schmittgen, T. D. 2001. Analysis of relative gene expression data using real-time quantitative PCR and the 2(T)(-Delta Delta C) method. Methods 25:402-408.

Maier, F. J., Miedaner, T., Hadeler, B., Felk, A., Salomon, S., Lemmens, M., Kassner, H., and Schafer, W. 2006. Involvement of trichothecenes in fusarioses of wheat, barley and maize evaluated by gene disruption of the trichodiene synthase (Tri5) gene in three field isolates of different chemotype and virulence. Mol. Plant Pathol. 7:449-461.

Mehrabi, R., Ding, S., and Xu, J. R. 2008. MADS-box transcription factor Mig1 is required for infectious growth in Magnaporthe grisea. Eukaryot. Cell 7:791-799.

Mewes, H. W., Frishman, D., Güldener, U., Mannhaupt, G., Mayer, K., Mokrejs, M., Morgenstern, B., Münsterkötter, M., Rudd, S., and Weil, B. 2002. MIPS: A database for genomes and protein sequences. Nucleic Acids Res. 30.

Mitter, V., Francl, L. J., Ali, S., Simpfendorfer, S., and Chakraborty, S. 2006. Ascosporic and conidial inoculum of Gibberella zeae play different roles in Fusarium head blight and crown rot of wheat in Australia and the USA. Aust. Plant Pathol. 35:441-452.

Palmer, J. M., Perrin, R. M., Dagenais, T. R. T., and Keller, N. P. 2008. H3K9 methylation regulates growth and development in Aspergillus fumigatus. Eukaryot. Cell 7:2052-2060.

Proctor, R. H., Hohn, T. M., and McCormick, S. P. 1995. Reduced virulence of Gibberella zeae caused by disruption of a trichothecene toxin biosynthetic gene. Mol. Plant-Microbe Interact. 8:593-601.

Proctor, R. H., Hohn, T. M., and McCormick, S. P. 1997. Restoration of wild-type virulence to Tri5 disruption mutants of Gibberella zeae via gene reversion and mutant complementation. Microbiology 143:25832591 .

Seong, K., Li, L., Hou, Z. M., Tracy, M., Kistler, H. C., and Xu, J. R. 2006. Cryptic promoter activity in the coding region of the HMG-CoA rediactase gene in Fusarium graminearum. Fungal Genet. Biol. 43:3441.

Todd, R. B., Murphy, R. L., Martin, H. M., Sharp, J. A., Davis, M. A., Katz, M. E., and Hynes, M. J. 1997. The acetate regulatory gene facB of Aspergillus nidulans encodes a $\mathrm{Zn}$ (II)2Cys6 transcriptional activator. Mol. Gen. Genet. 254:495-504

Trail, F., Gaffoor, I., and Vogel, S. 2005. Ejection mechanics and trajectory of the ascospores of Gibberella zeae (anamorph Fusarium graminearum). Fungal Genet. Biol. 42:528-533. 
Tribus, M., Galehr, J., Trojer, P., Brosch, G., Loidl, P., Marx, F., Haas, H. and Graessle, S. 2005. HdaA, a major class 2 histone deacetylase of Aspergillus nidulans, affects growth under conditions of oxidative stress. Eukaryot. Cell 4:1736-1745.

Tribus, M., Bauer, I., Galehr, J., Rieser, G., Trojer, P., Brosch, G., Loidl, P., Haas, H., and Graessle, S. 2010. A novel motif in fungal class 1 histone deacetylases is essential for growth and development of Aspergillus. Mol. Biol. Cell 21:345-353.

Urban, M., Bhargava, T., and Hamer, J. E. 1999. An ATP-driven efflux pump is a novel pathogenicity factor in rice blast disease. EMBO (Eur Mol. Biol. Organ.) J. 18:512-521.

Wang, A., Kurdistani, S.K., and Grunstein, M. 2002. Requirement of Hos2 histone deacetylase for gene activity in yeast. Science 298:1412-1414.

Yang, X., and Grégoire, S. 2005. Class II histone deacetylases: From sequence to function, regulation, and clinical implication. Mol. Cell. Biol. 25:2873-2884.

Zhou, X. Y., Heyer, C., Choi, Y. E., Mehrabi, R., and Xu, J. R. 2010. The CID1 cyclin C-like gene is important for plant infection in Fusarium graminearum. Fungal Genet. Biol. 47:143-151.

AUTHOR-RECOMMENDED INTERNET RESOURCE

Plant Expression Database (PlexDB): www.plexdb.org 